Cómo citar este artículo: Mora-Mendoza, E. Y., Sarmiento-Santos, A., \& Casallas-Caicedo, F. M. (2020). La influencia del tiempo de residencia en la conversión del dióxido de carbono en un sistema de descargas de barrera dieléctrica. Rev.investig.desarro.innov., 11 (1), $173-183$.

\title{
Influencia del tiempo de residencia en la conversión del dióxido de carbono en un sistema de descargas de barrera dieléctrica
}

\author{
Influence of residence time on the conversion of carbon \\ dioxide IN a dielectric barrier discharge system
}

\author{
Eduin Yesid Mora-Mendoza' \\ Armando Sarmiento-Santos ${ }^{2}$ \\ Francy Mayoli Casallas-Caicedo ${ }^{3}$
}

Recibido: octubre 15 de 2019

Aceptado: marzo 05 de 2020

\section{Resumen}

Se presenta un estudio sobre la importancia del tiempo de residencia, también llamado de permanencia, de un gas dentro de un gap de descarga de barrera de dieléctrico, para el tratamiento del dióxido de carbono $\mathrm{CO}_{2^{\prime}}$ el cual es un gas contaminante. Junto al $\mathrm{CO}_{2}$, se adicionó hidrógeno $\mathrm{H}_{2}$ para conformar la atmósfera de reacción de la descarga. Se estudió el comportamiento de la potencia entregada al sistema en función de la tensión de operación, utilizando alúmina y cuarzo como dieléctricos. También se estudió la conversión del $\mathrm{CO}_{2}$ en función del tiempo de residencia para tres diferentes composiciones de la mezcla $\mathrm{CO}_{2}+\mathrm{H}_{2}$ para tres diferentes valores de potencia eléctrica activa suministrada al sistema y para tres diferentes valores de frecuencia de operación, utilizando alúmina como dieléctrico. El porcentaje de conversión del $\mathrm{CO}_{2}$ en las condiciones de trabajo fijadas, se incrementó al aumentar el tiempo de residencia, independientemente de la composición de la mezcla de los dos gases, de la potencia eléctrica y de la frecuencia de operación, encontrando los valores más altos para la menor cantidad de $\mathrm{CO}_{2}$ en la mezcla, para la mayor potencia suministrada y para la menor frecuencia de operación.

Palabras clave: tiempo de residencia, descarga de barrera de dieléctrico, conversión del $\mathrm{CO}_{2}$, potencia eléctrica, frecuencia de resonancia.

\begin{abstract}
This paper presents a study about the importance of residence time, also called permanence time, of a gas in a gap of dielectric barrier discharge, for the treatment of carbon dioxide $\mathrm{CO}_{2}$, which is a pollutant gas. Along with $\mathrm{CO}_{2}$, hydrogen $\mathrm{H}_{2}$ is added to form the atmosphere of the discharge reaction. It was studied the behavior of power deliver to system as a function of operation voltage using alumina and quartz as dielectrics. $\mathrm{CO}_{2}$ conversion was studied as a function of residence time for three different compositions of the $\mathrm{CO}_{2}+\mathrm{H}_{2}$ mixture, for three different values of the active electrical power supplied to the system and for three different values of frequency of operation using alumina as dielectric. The percent conversion of $\mathrm{CO}_{2}$ in fixed working conditions was increased with increasing residence time, regardless of the composition of the mixture of the two gases, of the electrical power and the operation frequency, finding the highest values for the least amount of $\mathrm{CO}_{2}$ in the mixture, for higher power delivered and for the lower frequency of operation.
\end{abstract}

Keywords: residence time, Dielectric Barrier Discharge, $\mathrm{CO}_{2}$ conversion, electrical power, resonance frequency.

1 Ingeniero Electromecánico, Doctor en Ingeniería y Ciencia de los Materiales, Universidad Pedagógica y Tecnológica de Colombia, Duitama, Colombia. E-mail: eduin.mora@uptc.edu.co. ORCID: 0000-0002-8578-2990

2 Físico, Doctor en Ciencias e Ingeniería de los Materiales, Universidad Pedagógica y Tecnológica de Colombia, Tunja, Colombia. E-mail: asarmiento.santos@uptc.edu.co. ORCID: 0000-0003-3382-0104

3 Ingeniera Electromecánica, Magíster en Metalurgia y Ciencias de los Materiales, Universidad Pedagógica y Tecnológica de Colombia, Duitama, Colombia. E-mail: francy.casallas@uptc.edu.co. ORCID: 0000-0001-5559-3729 


\section{Introducción}

El uso del carbón, el petróleo y del gas natural, generó una era de prosperidad y de avance en el desarrollo humano sin precedentes, principalmente en los dos últimos siglos. El mundo todavía depende fuertemente de los combustibles fósiles, los cuales cubren cerca del $80 \%$ de sus necesidades energéticas (Olah \& Prakash, 2009). El incremento de las emisiones de $\mathrm{CO}_{2}$ contribuye al incremento de la temperatura global y el cambio climático, debido al efecto invernadero. Existen tres posibles estrategias para reducir las emisiones: reducir las cantidades emitidas de $\mathrm{CO}_{2}$ almacenarlo y reutilizarlo (Mikkelsen, Jorgensen \& Krebs, 2010; Hongqun, 2008). La última opción contempla mezclar el $\mathrm{CO}_{2}$ junto con $\mathrm{H}_{2}$, bajo ciertas condiciones, para obtener algunos subproductos, que pueden servir en diferentes procesos. Las nuevas especies creadas a partir de la hidrogenación del $\mathrm{CO}_{2}$, pueden ser: el metanol, metano, agua, monóxido de carbono y algunos otros hidrocarburos, que pueden ser excelentes combustibles, utilizados en motores de combustión interna; además son de fácil almacenaje y transporte (Wei-Wang, et al, 2011). La clave para la hidrogenación del $\mathrm{CO}_{2}$, es la disponibilidad del hidrógeno, pues se considera como una fuente de energía muy importante para los años venideros. Un catalizador de dióxido de zirconio y niquel operando a temperaturas de cerca de los $500 \mathrm{~K}$, puede hacer reaccionar los dos gases formando metanol y agua (Schild, 1991).

Los subproductos anteriores también se pueden obtener junto con metano, a través de una Descarga de Barrera de Dieléctrico (DBD). Un arreglo DBD consta de una fuente de alta tensión, la cual genera tensiones del órden de los kilovoltios, y frecuencias que van de los $500 \mathrm{~Hz}$ hasta 500 $\mathrm{kHz}$, desde la cual se realizan descargas eléctricas en un reactor. Este último está compuesto por una barrera dieléctrica, que puede ser de: alúmina $\left(\mathrm{Al}_{2} \mathrm{O}_{3}\right)$, vidrio pyrex, comunmente conocido como borosilicato o también de cuarzo; también se utilizan electrodos de cobre o acero inoxidable, normalmente conformados en estructuras cilíndricas (Mora-Mendoza, Sarmiento-Santos \& Casallas-Caicedo, 2014). Los gases en tratamiento entran al reactor, donde son sometidos a las descargas eléctricas que facilitan las reacciones químicas y con ello la generación de nuevas especies. Diferentes autores han trabajado con este tipo de configuraciones en el tratamiento de gases (Tae \& Wong, 2012; Tao, 2002; Sentek, 2009; Sarmiento, 2007; Eliasson, 1992).

Entre los parámetros que caracterizan las descargas y que influyen en la transformación de los gases, como el $\mathrm{CO}_{2^{\prime}}$ están: la tensión, la frecuencia, la potencia, la composición del gas y el tiempo de residencia. Este último representa el tiempo en que las moléculas del gas permanecen dentro del volumen de descarga del reactor. Algunos investigadores han realizado el estudio de la influencia de este importante parámetro en la conversión de gases (Indarto, 2007; Sazal \& Kundu, 2011).

En esta investigación, se evidencia la importancia del tiempo de residencia en el tratamiento del dióxido de carbono $\left(\mathrm{CO}_{2}\right)$, en presencia de hidrógeno $\left(\mathrm{H}_{2}\right)$, en un arreglo DBD que utilizó la alúmina como material dieléctrico, debido a sus buenas propiedades dieléctricas. Esto último a diferencia de lo reportado en la literatura, donde se presentan investigaciones en las que se utiliza principalmente cuarzo. La conversión del $\mathrm{CO}_{2}$ en función del tiempo de residencia, se estableció para tres variables, a saber: la composición de la mezcla $\mathrm{CO}_{2}+\mathrm{H}_{2}$ la potencia eléctrica activa suministrada al sistema y la frecuencia de operación.

\section{Materiales y métodos}

\subsection{El arreglo DBD}

El esquema del arreglo DBD empleado, se muestra en la figura 1. Luego se describen cada uno de sus componentes. 


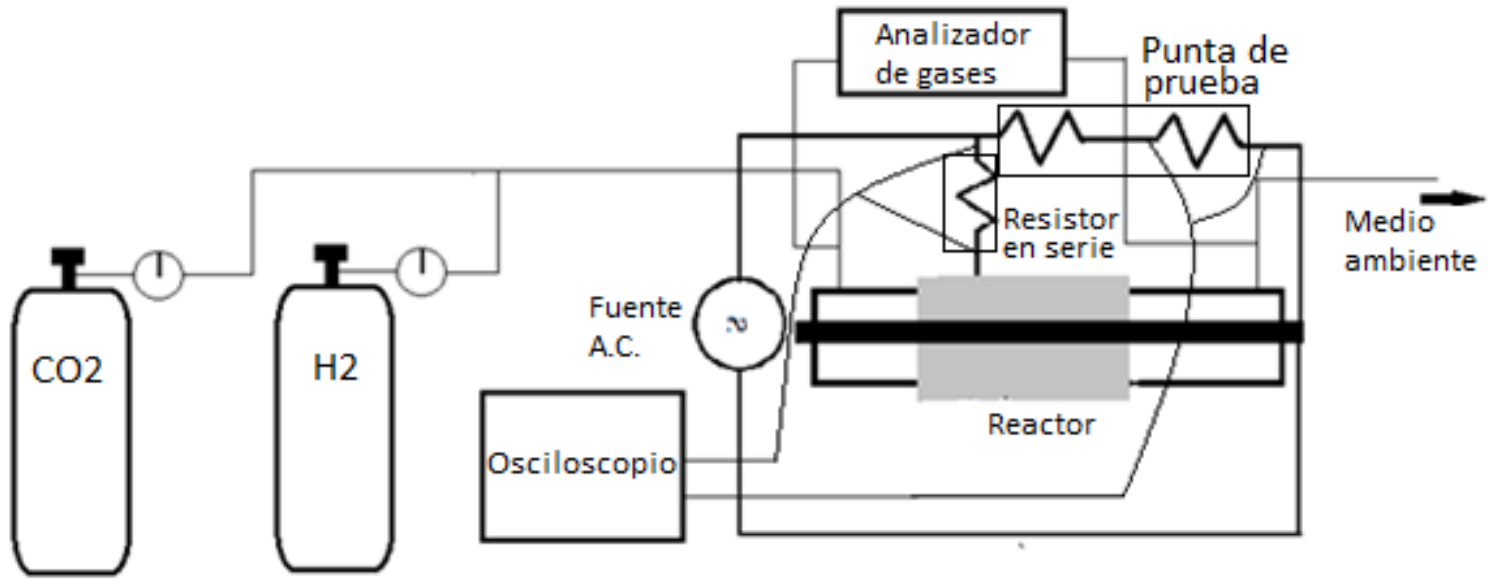

Figura 1. Esquema del montaje experimental utilizado para el tratamiento de $\mathrm{CO}_{2}$ mediante la DBD.

Reactor

El reactor utilizado para generar la DBD tiene una configuración cilíndrica, cuyo dieléctrico, que hace las veces de cámara del reactor, consta de un tubo de alúmina de $99,5 \%$ de pureza. El electrodo externo que recubre el dieléctrico, se fabricó de una lámina de acero inoxidable, con una longitud de $110 \mathrm{~mm}$, cumpliendo la función de electrodo de tierra. Como electrodo interno se utilizó una varilla de $5 / 8^{\prime \prime}$ de acero inoxidable, la cual fue sometida a remoción de óxidos y a un proceso de cromado electroquímico durante 20 minutos; este último proceso, permite recubrir toda la superficie de la varilla con este metal, el cual mejora las condiciones para la reacción dentro de la cámara.

El tubo de alúmina tiene unas dimensiones físicas de: $215 \mathrm{~mm}$ de largo, $30 \mathrm{~mm}$ de diámetro exterior y $2 \mathrm{~mm}$ de espesor. Estas dimensiones permiten un volumen de descarga para el gas de $36,67 \mathrm{ml}$. El reactor cuenta con dos elementos que se adaptan en los costados laterales del dieléctrico, teniendo como propósito, el acople de la línea de gases con la cámara del reactor; lo anterior es llevado a cabo mediante acoples rápidos. Estas tapas se fabricaron en teflón, material que tiene la propiedad de soportar las temperaturas que se generan durante la descarga.
Sistema de gases

El $\mathrm{CO}_{2}$ y el $\mathrm{H}_{2}$, con grado de pureza de $99,5 \%$, alimentan el reactor desde dos líneas provenientes de los cilindros; en cada línea se adaptó un regulador de presión y un medidor de flujo. Para la medición de la cantidad de $\mathrm{CO}_{2}$ antes y después de la descarga, en porcentaje de volumen en el gas, se utilizó el analizador de gases CM 0006 (CO2 Meter) el cual mide la concentración de $\mathrm{CO}_{2}$ en el gas de 0 a $100 \%$, utilizando tecnología de infrarrojo no dispersivo. Este instrumento se lleva a lectura de $0.0 \%$ vol, al inicio de cada prueba.

\section{Fuente de alimentación y sistema eléctrico}

Para la generación de plasma con la DBD, se necesitan señales de alta tensión y alta frecuencia. La fuente utilizada es la PWM500/DRIVE10 (Information Unlimited). Esta fuente proporciona tensiones que van de 0 a $40 \mathrm{kV}$ pico a pico; las frecuencias se pueden ajustar entre valores de 20 a $60 \mathrm{kHz}$ por medio de un potenciómetro, encontrando finalmente el valor requerido con la ayuda del osciloscopio. Para monitorear el comportamiento de las magnitudes eléctricas de: tensión, corriente y frecuencia, se utilizó un osciloscopio TBS 1062 (Tektronix), el cual permite un ancho de banda de $60 \mathrm{MHz}$, cuenta con dos canales, uno de los cuales se utilizó para monitorear el comportamiento de la 
tensión y el otro para el de la corriente. Desde este instrumento se puede verificar el desfase entre las señales debido al efecto inductivo de la fuente y al efecto capacitivo integrado del dieléctrico y del gas.

Para cuantificar el consumo total de la potencia eléctrica activa, se utilizó un medidor digital INTERTEK GS CAT II, el cual maneja un rango de potencia de 0 a $1800 \mathrm{~W}$. Este instrumento maneja dos conectores, uno en la parte posterior (macho) que lo acopla a la red de alimentación monofásica, y otro en la parte frontal (hembra), que permite la conexión del cable de alimentación de la fuente de poder. Para monitorear la señal de tensión de alimentación en el reactor, la cual es del orden de los kilovoltios, se utilizó una punta de prueba de alta tensión en una relación en la medición de 1000:1, con el fin de reducir el nivel de tensión a lo permitido en el osciloscopio. Para el monitoreo de la señal de corriente eléctrica, se conectó una resistencia de $100 \Omega$ en serie con el circuito del reactor, la cual está finalmente conectada al electrodo de tierra.

\subsection{Parámetros investigados}

Tiempo de residencia. El tiempo de residencia del gas dentro del reactor, se calculó mediante la ecuación 1.

$$
t=V d / Q g(s)
$$

Dónde:

$$
\begin{aligned}
& V d=\text { Volumen de descarga en el gap. } \\
& Q g=\text { Flujo del gas en } \mathrm{Nml} / \mathrm{min} .
\end{aligned}
$$

Los flujos de gas ajustados, fueron de 52, 74 y 96 $\mathrm{Nml} / \mathrm{min}$. Las condiciones nominales se consiguieron considerando $550 \mathrm{mmHg}$. La presión atmosférica y la temperatura promedio del ambiente donde se realizó la experimentación, fue de 291,15 K. Con los flujos de gas indicados, se consiguieron tiempos de residencia de 22,9, 29,7 y 42,3 segundos, respectivamente.

Conversión del $\mathrm{CO}_{2}$. Este parámetro indica la transformación o proporción de cambio del $\mathrm{CO}_{2}$ en otros compuestos o elementos, es decir:

$$
(\%) \mathrm{CO}_{2}=\frac{\text { cantidad de } \mathrm{CO}_{2} \text { convertido }}{\text { cantidad de } \mathrm{CO}_{2} \text { introducido }} * 100 \text { (\%) }
$$

La cantidad de $\mathrm{CO}_{2}$ introducido, es la que se mide sin descarga eléctrica a la salida del reactor. La cantidad de $\mathrm{CO}_{2}$ convertido, se calcula restando la medición tomada a la salida del reactor después de la descarga a la tomada sin descarga.

Proporción de $\mathrm{CO}_{2}$ en la mezcla. El porcentaje de $\mathrm{CO}_{2}$ en la mezcla $\mathrm{H}_{2}+\mathrm{CO}_{2}$, se ajustó a valores 10 , 20 y 40\%, a través de las lecturas dadas desde los rotámetros, manteniendo constantes los valores de potencia eléctrica suministrada al sistema y la frecuencia de operación en 105 W y 19,72 kHz, respectivamente.

Potencia eléctrica. La potencia suministrada al sistema tomó valores de 80,100 y $110 \mathrm{~W}$, manteniendo constante tanto el porcentaje de $\mathrm{CO}_{2}$ en la mezcla $\mathrm{H}_{2}+\mathrm{CO}_{2}$, así como la frecuencia, en $20 \%$ y $19,72 \mathrm{kHz}$, respectivamente.

Frecuencia eléctrica de operación. Para el ajuste de este parámetro, se mantiene constante tanto la tensión eléctrica en valor rms y el porcentaje de $\mathrm{CO}_{2}$ en la mezcla $\mathrm{H}_{2}+\mathrm{CO}_{2^{\prime}}$ en $13,15 \mathrm{kV}$ y $20 \%$, respectivamente; esto ajustando desde la fuente, frecuencias de 20, 21 y $25 \mathrm{kHz}$.

\section{Resultados y discusión}

3.1 Potencia suministrada en función de la tensión eléctrica, para alúmina y cuarzo

Debido a que la constante dieléctrica del material utilizado en los sistemas DBD, es un parámetro que afecta de manera importante el tratamiento del $\mathrm{CO}_{2}$ (Duan, 2015), se realizaron ensayos para 
estudiar el comportamiento de la potencia en función de la tensión aplicada al reactor, usando alúmina y cuarzo. El valor de potencia suministrada se toma 5 minutos después de iniciada la descarga. Las condiciones en el sistema, son: flujo de $74 \mathrm{Nml} / \mathrm{min}$, frecuencia de $19,72 \mathrm{kHz}$ y proporción en la mezcla de $80 \%$ de $\mathrm{H}_{2}$ y $20 \% \mathrm{CO}_{2}$.

En la figura 2, se presentan gráficamente los resultados del comportamiento, a frecuencia eléctrica de $19,72 \mathrm{KHz}$, flujo de $74 \mathrm{Nml} / \mathrm{min}$ y proporción en la mezcla de $80 \mathrm{H}_{2}$ y $20 \% \mathrm{CO}_{2}$. Es evidente que para la misma tensión de operación, la potencia suministrada al reactor de alúmina es más alta que la correspondiente al cuarzo, debido a la mayor capacitancia de la alúmina, lo que se traduce en mayor paso de corriente. Desde otro punto de vista, para disipar la misma potencia en los dos materiales se necesita un ajuste en la tensión más alto utilizando el reactor de cuarzo; por ejemplo, para disipar cerca de $98 \mathrm{~W}$ en la cámara de cuarzo se necesita una tensión de $12,72 \mathrm{kV}$, lo que en la cámara de alúmina se conseguirá a $12,01 \mathrm{kV}$, en 5 minutos de descarga, permitiendo manejar en la alúmina corrientes más altas, es decir mayor actividad en el reactor y por consiguiente mayor transformación. Debido a esto, se utilizó la alúmina como material dieléctrico en esta investigación.

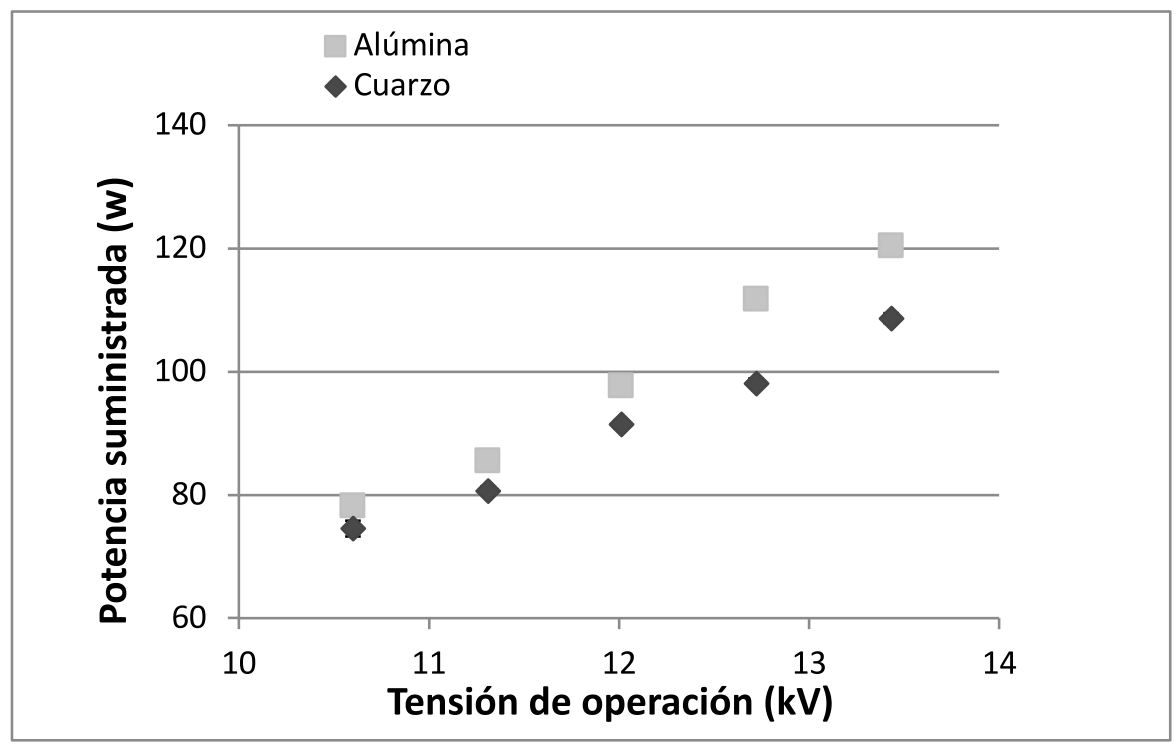

Figura 2. Potencia suministrada al sistema en función de la tensión de operación, para dieléctricos de alúmina y cuarzo.

\subsection{2 Caracterización de la descarga}

Las DBD, se dividen en las descargas filamentares y las difusas $u$ homogéneas; en apariencia, las descargas homogéneas se distribuyen uniformemente a través de todo el volumen de descarga. En contraste, las descargas filamentares consisten en un conjunto de canales de descarga discretos aleatoriamente distribuidos (Massines, 2005).

Se alimentó el reactor con los gases de estudio, es decir, dióxido de carbono $\mathrm{CO}_{2}$, e hidrógeno $\mathrm{H}_{2}$.
Inicialmente, se introduce la mezcla en proporción 1:1 con un flujo total de $74 \mathrm{Nml} / \mathrm{min}$. Se incrementó la potencia suministrada al sistema cambiando las señales de tensión y de frecuencia desde la fuente, hasta valores de $5,65 \mathrm{kV}$ y 19,72 $\mathrm{kHz}$, respectivamente. En estas condiciones se presenta la descarga filamentar, la cual se evidencia con la distorsión de la señal de corriente (presenta la menor amplitud), pudiéndose apreciar ésta junto con la señal de tensión (presenta la mayor amplitud), en el osciloscopio, como se aprecia en la figura 3. 


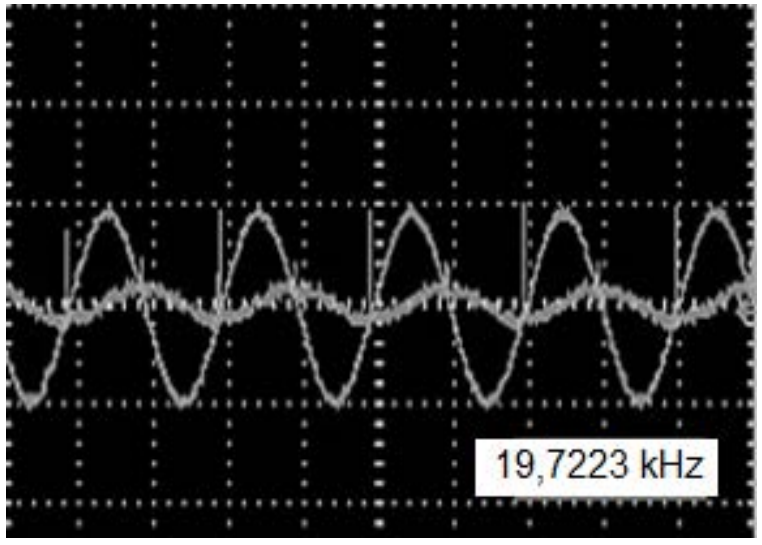

Figura 3. Señales de tensión y corriente en el reactor a $19,72 \mathrm{KHz}$, con alúmina como dieléctrico.

Las señales de tensión y corriente muestran que existe un adelanto por parte de la señal de tensión, lo que evidencia que para la frecuencia de operación de 19,72 KHz, el circuito del sistema presenta predominancia inductiva, esto debido a que la reactancia inductiva de acople de la fuente de poder, tiene más valor que la reactancia capacitiva total del gas y del dieléctrico.

Se realizaron algunos ensayos buscando condiciones favorables para plasma estable. Un conjunto adecuado de valores para las variables, se consigue cuando la tensión es de 13,15 kV, la potencia suministrada de $105 \mathrm{~W}$, y la frecuencia de $19,69 \mathrm{kHz}$; en la figura 4 se muestra una imagen del comportamiento de las señales de tensión y corriente para estas condiciones, donde se aprecia una distorsión de las mismas debido a las reacciones químicas presentes dentro del reactor.

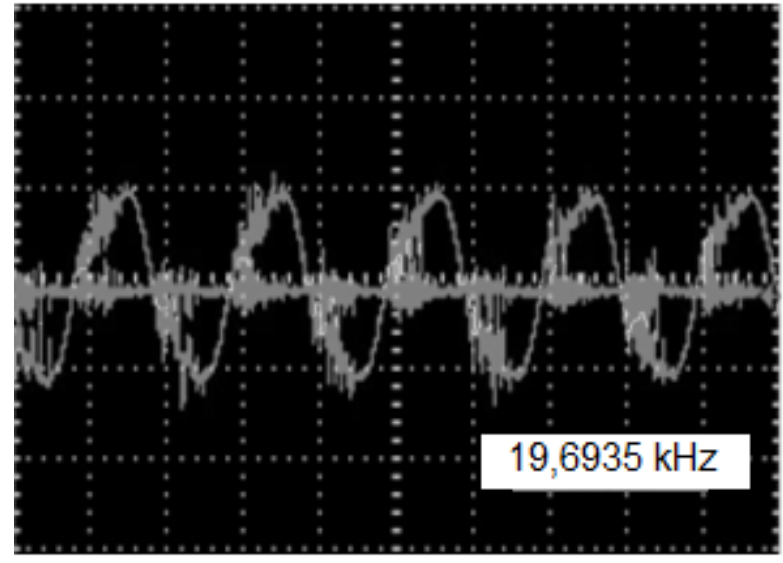

Figura 4. Señales de tensión y corriente para $50 \%$ de $\mathrm{CO}_{2}$, a $19,69 \mathrm{KHz}, 105 \mathrm{~W}$ y $13,15 \mathrm{kV}$, utilizando alúmina como dieléctrico.

3.3 Efectos del tiempo de residencia en la conversión del $\mathrm{CO}_{2}$

Composición en la mezcla. En la figura 5, se aprecia el comportamiento de la transformación del $\mathrm{CO}_{2}$ para los tres tiempos de residencia: 22,9 , 29,7 y $42,3 \mathrm{~s}$, en las tres composiciones de $\mathrm{CO}_{2}$ en la mezcla: 10, 20 y $40 \%$, con potencia suministrada de $105 \mathrm{~W}$ y frecuencia de operación de $19,72 \mathrm{kHz}$.

Se evidencia que, independientemente de la composición en la mezcla, la transformación del $\mathrm{CO}_{2}$ se incrementa para tiempos de residencia mayores, alcanzando su máximo valor en $43,71 \%$ para un tiempo de residencia de 42,3 s y $10 \%$ de proporción de $\mathrm{CO}_{2}$ en la mezcla. Lo anterior sugiere que entre más tiempo permanezcan las moléculas de $\mathrm{CO}_{2}$ y $\mathrm{H}_{2}$ dentro del volumen de descarga, mayor oportunidad se tiene de reaccionar en el gap para así formar otros compuestos. Visto de otra manera, para obtener el mismo nivel de conversión en dos composiciones diferentes, es necesario mantener por más tiempo dentro del reactor el gas que contiene mayor cantidad de $\mathrm{CO}_{2}$ en la composición. 


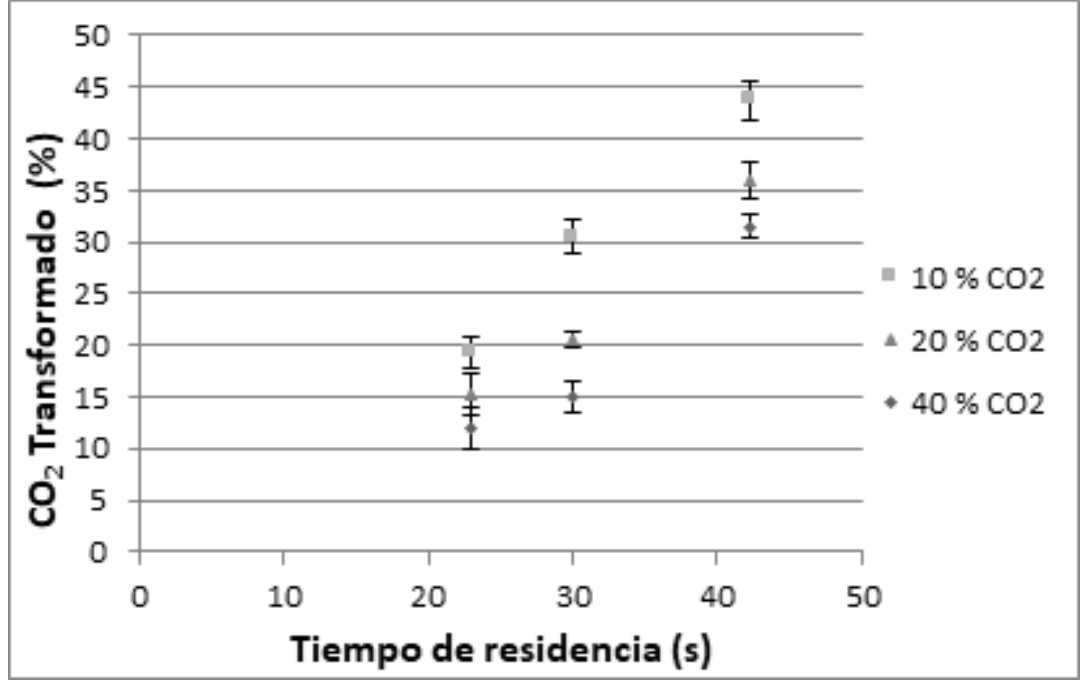

Figura 5. Porcentaje de $\mathrm{CO}_{2}$ transformado en función del tiempo de residencia, para tres concentraciones, a frecuencia de $19,72 \mathrm{kHz}$ y potencia de $105 \mathrm{~W}$, usando alúmina.

Igualmente, se deduce de la figura 5, que para el mismo tiempo de residencia, es decir, el mismo flujo, las mejores transformaciones se presentan cuando el contenido de $\mathrm{CO}_{2}$ en la mezcla, se hace menor; esto implica un consumo de $\mathrm{H}_{2}$ elevado. A pesar de lo anterior, con los porcentajes de transformación obtenidos y para los tiempos de residencia fijados, se hace evidente que el tiempo de residencia es un parámetro importante en la transformación del $\mathrm{CO}_{2}$, siendo este relativamente fácil de fijar, con base en las condiciones geométricas del reactor y en el flujo de alimentación en los rotámetros.

Potencia eléctrica. En la figura 6 , se aprecia el comportamiento de la transformación en los tres tiempos de residencia estudiados, para tres valores de potencia: 80,100 y $110 \mathrm{~W}$, frecuencia de operación de $19,72 \mathrm{kHz}$ y composición en la mezcla de $80 \% \mathrm{H}_{2}$ y $20 \% \mathrm{CO}_{2}$.
La transformación del $\mathrm{CO}_{2}$ se incrementa al aumentar el tiempo de residencia, alcanzando su máximo valor en $33.38 \%$ para un tiempo de residencia de 42,3 s y $110 \mathrm{w}$ de potencia aplicada; es decir, en condiciones de mayor cantidad de energía durante el mayor tiempo posible. El incremento de la potencia de entrada, permite suministrar más energía para disociar las moléculas y con ello generar más electrones energéticos, los cuales permiten fragmentar otras moléculas (Hyung, 2004). La conversión es de $17,54 \%$ y de $33,38 \%$, para tiempos de residencia de 22,9 y $42,3 \mathrm{~s}$, respectivamente, a potencia suministrada de 110 $\mathrm{W}$, interpretando de lo anterior, que la transformación se duplica aproximadamente al duplicar también el tiempo de residencia, para la misma potencia. Comportamiento similar se presentó en las otras potencias estudiadas. 


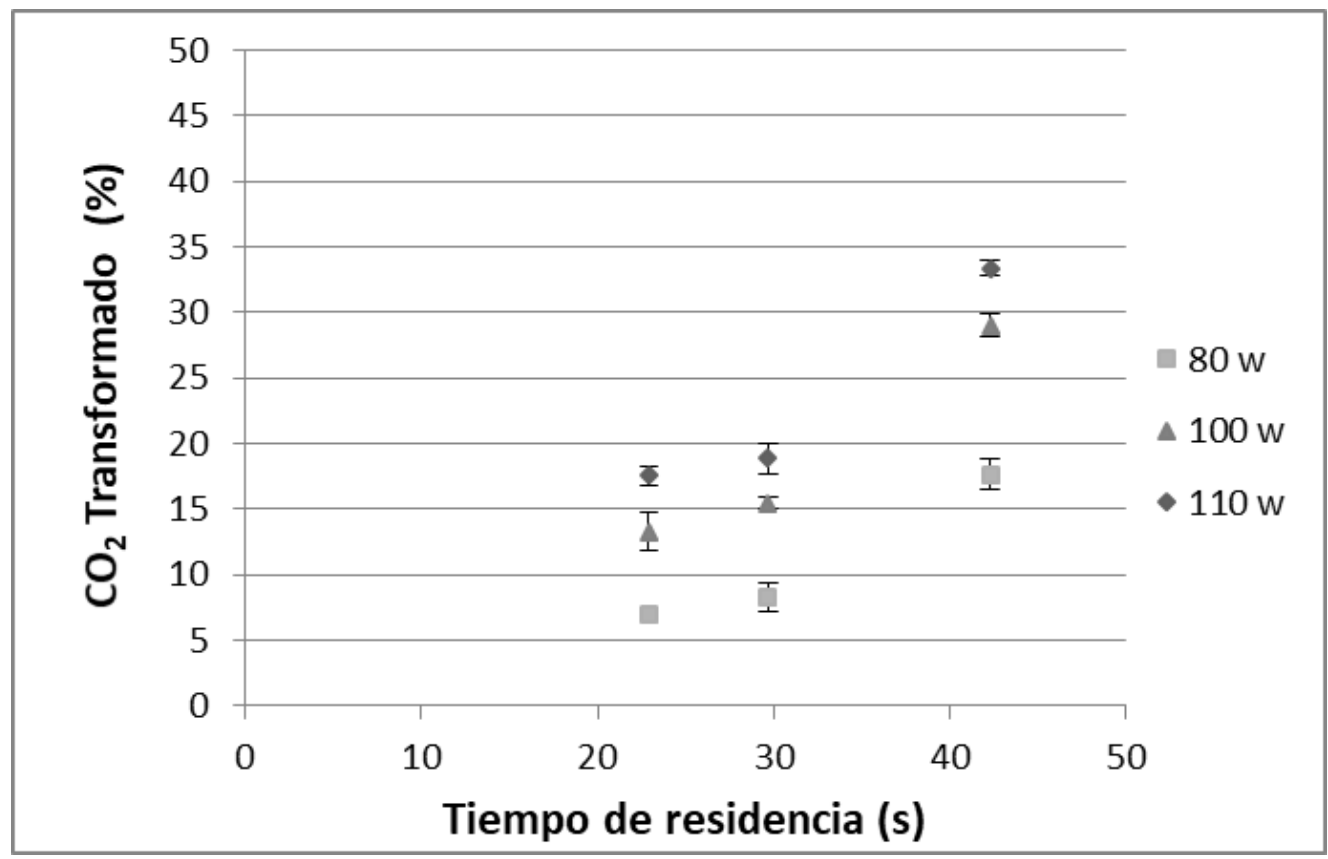

Figura 6. Porcentaje de $\mathrm{CO}_{2}$ transformado en función del tiempo de residencia, para tres diferentes potencias, a 19,72 kHz y composición en la mezcla de $80 \% \mathrm{H}_{2}$ y $20 \% \mathrm{CO}_{2}$, usando alúmina.

En este caso se infiere que, para el mismo gasto energético, mayor formación de nuevos productos o mayor cantidad de los ya existentes se dará en la medida que las moléculas permanezcan más tiempo dentro del reactor; lo anterior sugiere una vez más, que se justifica el desarrollo de reactores que permitan aumentar el tiempo de residencia de las moléculas dentro de los mismos.

Frecuencia eléctrica de operación. En la figura 7, se presenta el comportamiento del $\mathrm{CO}_{2}$ transformado, en función del tiempo de residencia, para tres valores de frecuencia: 20,21 y $25 \mathrm{kHz}$, con tensión de operación de 13,15kV y composición en la mezcla de $80 \% \mathrm{H}_{2}$ y $20 \% \mathrm{CO} 2$. En las tres frecuencias estudiadas, la transformación del
$\mathrm{CO}_{2}$, aumenta con el correspondiente aumento del tiempo de residencia, alcanzando su máximo valor en $31,04 \%$, para un tiempo de residencia de 42,3 s y $20 \mathrm{kHz}$ de frecuencia de operación.

La conversión es de 13,64\% y 19,63\%, para tiempos de residencia de 22,9 y 29,7 s, respectivamente, en la misma frecuencia de los $20 \mathrm{kHz}$. En esta misma frecuencia, el cambio en la transformación es de $17,4 \%$, pasando de 13,64 a $31,04 \%$ al aumentar el tiempo de residencia en 19,4 s, lo cual demuestra nuevamente la importancia de este parámetro en el proceso de transformación. Comportamientos similares se presentan en las otras frecuencias, como se aprecia en la figura 7. 


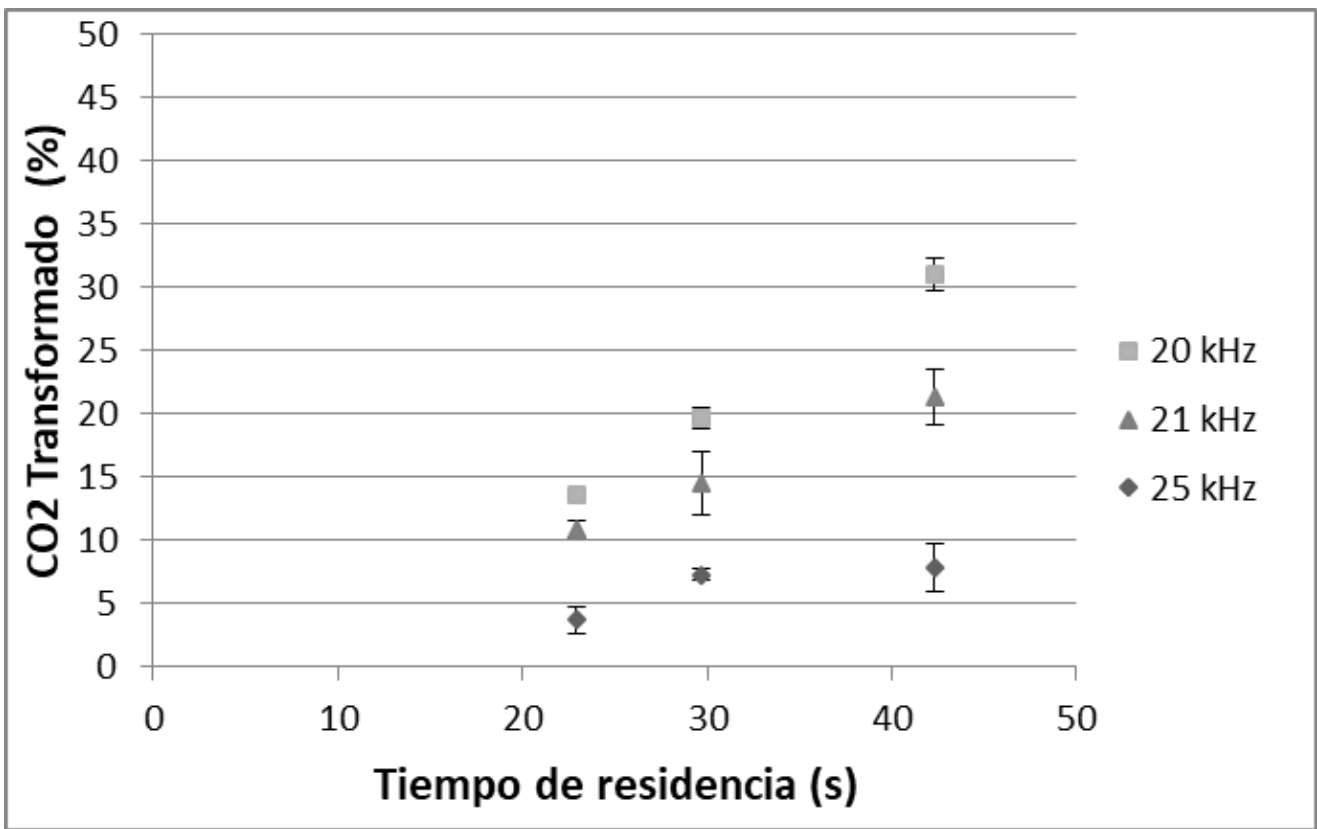

Figura 7. Porcentaje de $\mathrm{CO} 2$ transformado en función del tiempo de residencia, para tres frecuencias, a tensión de 13,15 kV y composición en la mezcla de $80 \%$ H2 y $20 \%$ CO2, usando alúmina.

La impedancia total en el circuito, está determinada por la impedancia de acople de la fuente y por la resistencia y capacitancia del reactor (incluyendo dieléctrico, gas y electrodos). Cuando la frecuencia de operación hace que se aproxime al punto de resonancia, la impedancia total alcanza su valor mínimo. Al conseguir esta condición, la máxima demanda de potencia es obtenida, y con ello la mayor conversión de $\mathrm{CO}_{2}$.

Para frecuencias más altas y más bajas del punto de resonancia, el sistema DBD se comporta con predominancias inductivas y capacitivas, respectivamente (dado que en el inductor y el capacitor, la impedancia es directa e inversamente proporcional a la frecuencia, respectivamente), y en los dos casos la impedancia total aumenta (Chong-Lin Song, 2008), y con ella disminuye la conversión de $\mathrm{CO}_{2}$. Es evidente que en el sistema implementado la predominancia es inductiva, dado que al aumentar la frecuencia se disminuye la conversión para el mismo tiempo de residencia. En la figura 8, se muestra el comportamiento predominante de la impedancia a diferentes frecuencias, así como la zona de frecuencias que corresponde a la experimentación realizada. 


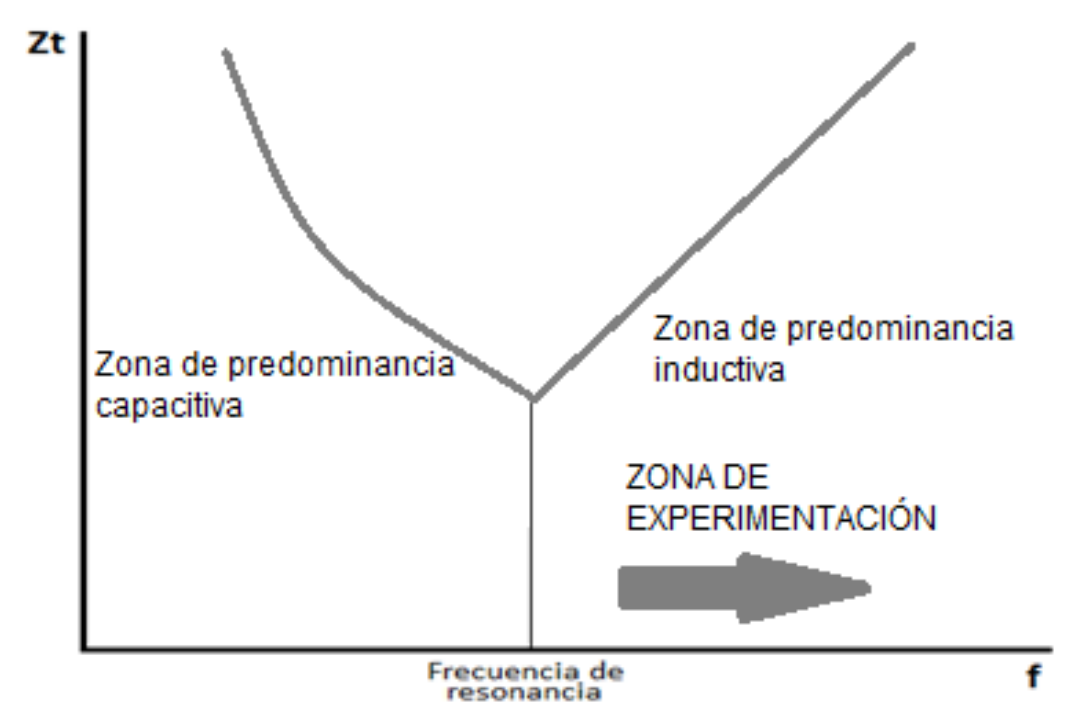

Figura 8. Impedancia del circuito en función de la frecuencia para el sistema DBD.

\section{Conclusiones}

Se estudió la conversión del $\mathrm{CO}_{2}$ en una DBD, en función del tiempo de residencia, en diferentes composiciones de la mezcla $\mathrm{CO}_{2}+\mathrm{H}_{2^{\prime}}$ para diferentes valores de potencia eléctrica y en diferentes valores de frecuencia de operación. En todos los casos estudiados, la conversión del $\mathrm{CO}_{2}$ aumentó de forma considerable con el aumento del tiempo de residencia, lo cual se consigue reduciendo el flujo de gas en el mismo volumen de descarga. Las señales eléctricas de tensión, corriente y frecuencia fueron monitoreadas, evidenciando el desfase entre las señales de tensión y corriente permitiendo así identificar que la impedancia de salida de la fuente tiene predominancia sobre las capacitancias del gas y del dieléctrico.

Independientemente de la composición de la mezcla $\mathrm{CO}_{2}+\mathrm{H}_{2}$, la transformación del $\mathrm{CO}_{2}$, se incrementa para tiempos de residencia mayores debido a que entre más tiempo permanezcan las moléculas de $\mathrm{CO}_{2}$ y $\mathrm{H}_{2}$ dentro del volumen de descarga, mayor oportunidad tienen de reaccionar; las mejores transformaciones se presentan cuando el contenido de $\mathrm{CO}_{2}$ en la mezcla se hace menor, implicando un consumo de $\mathrm{H}_{2}$ elevado. $\mathrm{La}$ transformación del $\mathrm{CO}_{2}$, se incrementa al aumentar el tiempo de residencia independientemente de la potencia aplicada. En el mismo gasto energético, mayor formación de nuevos productos o mayor cantidad de los ya existentes, se dará en la medida que las moléculas permanezcan más tiempo dentro del reactor.

La transformación del $\mathrm{CO}_{2}$, aumenta con el correspondiente aumento del tiempo de residencia en las tres frecuencias estudiadas. Los mejores niveles de transformación se consiguen con la menor frecuencia, dado que en el sistema implementado la predominancia es inductiva. La transformación del $\mathrm{CO}_{2}$, está por tanto determinada de manera importante por el punto de resonancia el cual depende de las características eléctricas del reactor y de la fuente. Con este estudio se demuestra que el tiempo de residencia es un parámetro importante en la transformación del $\mathrm{CO}_{2}$, siendo este fijado con el flujo de alimentación en los rotámetros; lo anterior permite contribuir al diseño de reactores más eficientes que permitan el óptimo tratamiento de este tipo de gases. 


\section{Referencias}

Chong-Lin Song. (2008). Simultaneous removals of NOx, HC and PM from diesel exhaust. Journal of Hazardous Materials, 166, 523-530.

Duan, X. (2015). Effect of dielectric Packing materials on the decomposition of carbon dioxide using DBD microplasma reactor. AIChE Journal, 61(3), 898- 903.

Eliasson, B. F. (1992.). Hydrogenation of C02 in a Silent Discharge. Corporate Research Report CRB-92-002 C.

Hongqun Yang, Z. X. (2008). Progress in carbon dioxide separation and capture: A review. Journal of Environmental Sciences, 20, 14-27.

Hyung Keun Song, J.-W. C.-K. (2004). Synthesis gas production via dielectric barrier discharge. Catalysis Today, 89, 27-33.

Indarto, A. (2007). Hydrogen production from methane in a dielectric barrier discharge. Journal of the Chinese Institute of Chemical Engineers, 39, 23-28.

Massines, N. G. (2005). Glow and Townsend dielectric barrier discharge in. Plasma Phys. Control. Fusion, 47, 577-588.

Mikkelsen, M. J., Jorgensen, M., \& Krebs., M. (2010). The teraton challenge. A review of fixation and transformation of carbon dioxide. Energy \& Environmental Science, 3 (1), 43-81.

Mora-Mendoza, E. Y., Sarmiento-Santos, A., \& Casallas-Caicedo, F. M. (2014). Implementación de un sistema de tratamiento con plasma para gases utilizando una celda de descarga de barrera de dieléctrico. Revista de investigación, desarrollo e innovación, 5 (1), 56-65. doi: https://doi. org/10.19053/20278306.3141
Olah, G., \& Prakash, G. (2009). Chemical Recycling of Carbon Dioxide to Methanol and Dimethyl Ether: From Greenhouse Gas to Renewable, Environmentally Carbon Neutral Fuels and Synthetic Hydrocarbons. The journal or organic C, 74 (1), 487-498.

Sarmiento, B. B. (2007). Hydrogen production by reforming of hydrocarbons and alcohols in a dielectric barrier discharge. (I. d.-U. Sevilla)., Ed.) Journal of Power Sources 169, 140-143.

Sazal, K., \& Kundu, E. M. (2011). Experimental investigation of alumina and quartz as dielectrics for a cylindrical. Chemical Engineering Journal, 180, 178-189.

Schild, C. W. (1991). Carbon dioxide hydrogenation over nickel/zirconia catalysts from amorphous precursors: on the mechanism of methane formation. The Journal of Physical Chemistry, 95 (16), 6341-6346.

Sentek, J. K. (2009). Plasma-catalytic methane conversion with carbon dioxide in dielectric. Applied Catalysis B: Environmental, 94, 19-26.

Tae Kyung Kim., \& Wong, G. (2012). Reaction between methane and carbon dioxide to produce syngas in dielectric. Journal of Industrial and Engineering Chemistry, 18, 1710-1714.

Tao Jiang, Y. (2002). Plasma methane conversion using dielectric-barrier. Catalysis Today, 72, 229-235.

Wei-Wang, S. W. (2011). Recent advances in catalytic hydrogenation of carbon dioxide. Chem Soc Rev, 40, 3703-3727. 
